\title{
Elementos constitucionales en la red global
}

\author{
Rafael Bustos Gisbert \\ Profesor titular de Derecho constitucional \\ Universidad de Salamanca
}

Recibido: 01.10 .2012

Aceptado: 15.10.2012

Resumen: La tesis principal sostenida en este artículo es que el diálogo judicial es un nuevo concepto o una nueva categoría que puede resolver algunos problemas propios de las situaciones de pluralismo constitucional. Pero no toda cita de una jurisdicción extranjera puede ser considerada una forma de diálogo. Los diálogos judiciales tienen sus propios objetivos, estructura y solución, diversos de los meros diálogos comparativos entre cortes.

Palabras clave: diálogos judiciales, pluralismo constitucional, superposiciones en la protección de derechos.

Abstract: The main thesis sustained in the paper is that judicial dialogue is a new concept/category that may solve some problems arising in situations of constitutional pluralism. But not every quotation of a foreign jurisdiction can be considered a form of dialogue. Judicial dialogues have their own objectives, structure and solution diverse from the mere comparative dialogues among courts.

Key words: judicial dialogues, constitutional plimalism, overlappings in the protection of human rights.

Sumario: 1. Pluralidad de Constituciones incompletas en un mundo global. - 2. Una red global de elementos constitucionales.-3. La resolución de conflictos en la red constitucional.-Bibliografía utilizada

\section{PLURALIDAD DE CONSTITUCIONES INCOMPLETAS EN UN MUNDO GLOBAL}

La nueva realidad global planea, desde el punto de vista de la Ciencia del Derecho Constitucional, la necesidad de adaptar las categorías y conceptos tradicionales de esta rama del conocimiento jurídico. No podemos hacer tabla rasa de los mismos, pues ello rompería la necesaria unidad discursiva e histórica de toda ciencia, pero quizás podamos reformular algunos conceptos y acuñar, a partir de ellos, otras categorías nuevas útiles para el análisis de la cambiante realidad de nuestros días.

Debemos, para ello, partir del concepto clave del Derecho Constitucional: la propia idea de Constitución. Este concepto se asienta, a su vez, en la concurrencia de un triple elemento. La Constitución es la norma jurídica suprema del ordenamiento de una comunidad (elemento formal: supremacía de la Constitución), acordada por un pueblo movilizado en momentos de ruptura 
histórica con el pasado (elemento constitutivo: expresión del poder constituyente), en el que se establecen las bases sustanciales de convivencia y los procedimientos de adopción de decisiones (elemento material: el viejo, pero vigente, art. 16 de la declaración de derechos de 1789).

¿Es este concepto aplicable a la realidad actual, en particular en ese vaporoso concepto llamado globalización? Para avanzar cualquier respuesta tentativa debemos, antes, tener en cuenta dos factores esenciales y complementarios.

En primer término los cambios operados en la premisa ontológica básica de la idea de Constitución. Nos referimos, obviamente, al Estado. En efecto, el Estado es el presupuesto de los tres elementos de la idea de Constitución apuntada. Es norma jurídica suprema del Estado, es expresión del poder constituyente del Estado y regula los derechos fundamentales y la división de poderes en el Estado. Y no cualquier tipo de Estado. Es el Estado propio de la Europa del Siglo XIX. Aunque no sepamos muy bien si realmente el Estado así considerado era el realmente vigente o, más bien, era una expresión idealizada; lo cierto es que la base institucional sobre la que se asienta la idea de Constitución es el Estado soberano tal y como es definido por la dogmática del derecho público y por la Teoría del Estado del siglo XIX y principios del XX: un Estado soberano capaz de resolver todos los problemas planteables en el ámbito jurídico, social, económico, militar, cultural, etc. Un ente que no reconoce poderes superiores ni inferiores susceptibles de limitar su poder y que se relaciona con el resto de Estados en posiciones de igualdad jurídica. Un ente, a la postre, soberano en los términos acuñados por BoDino.

Ese Estado (idealizado o no) se ha visto superado por la realidad. Ninguno de sus elementos definidores ha resistido la revolución tecnológica desde finales de los años 80 del pasado siglo. La soberanía no puede en ningún caso definirse en los mismos términos. Desde luego Está cuestionada (MACCORMICK), puede que el concepto esté en transición (WALKER) o, simplemente, que se haya convertido en postsoberanía. La idea de pueblo tampoco es ya unánime como han mostrado los debates constitucionales europeos (Tribunal Constitucional Federal, Grimm, Habermas o WeILER). Y algo parecido puede decirse respecto al concepto de territorio en una situación económica y tecnológica en que las fronteras han perdido gran parte de su significado. El viejo concepto de Estado ya no existe en la realidad. Cualquier análisis constitucional debe tener hoy en cuenta este dato. Y podemos intuir claramente que la transformación en la premisa ontológica del concepto de Constitución no puede sino tener consecuencias también transformadoras en el propio concepto madre de todo el Derecho Constitucional.

En segundo término, debemos también tener en cuenta que este fenómeno no es sólo europeo. Es universal. No podemos entender el Derecho constitucional hoy sin tener en cuenta este factor. Como tampoco podemos entender los procesos de integración regional (sean económicos, políticos, o una integración a través de los derechos fundamentales) sin tener en cuenta 
que la superación del Estado no sólo se produce como consecuencia de fenómenos de ámbito territorial continental, sino en un contexto que no conoce de naciones, pero tampoco de continentes por muy lejanos que se encuentren entre sí. Es un nuevo mundo en el que buena parte de los problemas planteados sólo pueden afrontarse desde instancias internacionales o desde la profunda interconexión entre centros de ejercicio del poder alejados sólo en el espacio, no en su interdependencia. Por ello una explicación de los cambios en el Derecho Constitucional actual no puede dejar de tener en cuenta el proceso universal de globalización.

Podríamos plantear que desaparecida la premisa ontológica de la idea de Constitución (el Estado) habría que declarar la defunción del propio concepto y con él del propio Derecho Constitucional. Pero no conviene olvidar que los conceptos y categorías jurídicas se crean al servicio de la convivencia entre seres humanos. Y que la idea de Constitución (y con ella de todos los conceptos propios del Derecho Constitucional) no sirven sino a la idea de limitación jurídica del poder que recibió el nombre de constitucionalismo y que probablemente hoy sea tan necesario defender como lo fue en los convulsos siglos XVII y XIX en Europa.

¿Puede existir un constitucionalismo sin Estado? Se preguntaba hace ya algunos años el profesor DíEz PicAzo. Desde luego lo que si ha de ocurrir es que este nuevo constitucionalismo requiere desprenderse (en la afortunada expresión de WALKER) de las lentes estatales. Esto es, eliminar del análisis constitucional aquellos elementos que son inseparables del concepto decimonónico de Estado. Ello nos dará una visión aparentemente distorsionada pues se separa de lo que habitualmente estábamos acostumbrados a observar y analizar desde el Derecho constitucional. Pero nos permitirá reconocer y aplicar conceptos más allá del Estado. Ciertamente los perfiles serán borrosos, pero esto es inevitable. Los conceptos y las categorías jurídicas adquieren tales perfiles nítidos después de un largo proceso que puede durar siglos. Además encontraremos importantes problemas de traducción (VON BOGDANDY) puesto que las palabras designarán realidades diferentes de manera que en cada caso deberemos traducir el lenguaje conceptual constitucional basado en la vieja idea de Estado a un nuevo lenguaje asentado en una situación superadora de tal Estado o, sin más, a realidades no-estatales.

Desprenderse de las lentes estatales no supone prescindir totalmente del concepto Estado en el análisis. Supone tan sólo que dejemos de observar los fenómenos constitucionales sólo desde la lógica del Estado,lo que no implica que la lógica de éste mantenga a menudo su sentido. Esto es importante recalcarlo porque no se trata de sustituir al Estado como célula básica en la articulación jurídico-constitucional de la nueva realidad. Supone situarle en su lugar adecuado en esa realidad. Lugar que sigue siendo esencial (véase infra), pero en el que ha perdido la posición de exclusividad característica del siglo XIX. Del mismo modo que los nuevos lugares desarrollan un discurso constitucional incompleto a través de metaconstituciones, el Estado, al atribuir a 
entes ajenos a su control el ejercicio de competencias esenciales derivadas de su antigua soberanía, también ha devenido un lugar incompleto pues ha perdido su carácter omnicomprensivo. Ello, en el plano de nuestro análisis significará que la constitución estatal se habrá, así, también convertido en metaconstitución pues no podrá regular todas las decisiones que afectan a sus ciudadanos, sino sólo aquellas que no haya transferido a otro lugar.

La situación descrita recibe el gráfico nombre de pluralismo constitucional (aunque en castellano es mucho más correcto el término de pluralidad de constituciones tal y como lo utiliza CRUZ) cuya definición más certera, a nuestro entender, es atribuible a uno de sus mejores teóricos, MACCORMICK: aquella situación en la que existen, al menos, dos constituciones, cada una reconocida como válida, pero sin que ninguna reconozca a la otra como fuente de su validez. Esto es, situaciones en las que existe una pluralidad de órdenes normativos institucionales, cada uno con una constitución, al menos en el sentido de cuerpo de normas de rango superior que establecen y condicionan el ejercicio del poder político y en el que se reconocen, mutuamente, legitimidad el uno al otro, pero sin que se afirme la supremacía de uno sobre otro. Ello supone alejarse del vicio fundacionalista que, a juicio de MACCORMICK, inspira buena parte del pensamiento filosófico desde fines del Siglo XVI y avanzar en la línea del reconocimiento de que nuestra existencia normativa está anclada en una variedad de sistemas institucionales.

Para empezar no nos queda más remedio que diferenciar distintos tipos de situaciones de pluralismo constitucional. Estas situaciones pueden, siguiendo a WALKER (2009) clasificarse en cuatro grandes grupos:

- Pluralismo constitucional por Incorporación Institucional en aquellos casos en que un sistema constitucional es incorporado al propio. Es a la que responde la UE, la Comunidad Andina y MERCOSUR.

- Pluralismo constitucional por «reconocimiento sistémico». No se trata de la aceptación de un ordenamiento por otro sino que se adoptan como propios elementos de otro sistema jurídico para definirse a sí mismo. Este sería el caso de los derechos humanos de ámbito transnacional; el derecho comercial transnacional y el derecho criminal transnacional.

- Pluralismo constitucional por «coordinación normativa»: se trata de una categoría vaga en la medida en que resulta extraordinariamente moderna e institucionalmente muy débil e incluye el efecto casi natural de confluencia entre ordenamientos jurídicos como consecuencia de la creación de sedes de encuentro y debate generadoras de directrices ampliamente compartidas por los diversos ordenamientos jurídicos. Este sería el caso de algunas agencias o cuerpos vinculados a Naciones Unidas como la OMS.

- Pluralismo constitucional por «consideración empática»: referido a situaciones en las que sin existencia de ninguno de los vínculos característicos de las situaciones de pluralismo constitucional (ni 
una incorporación institucional, ni un reconocimiento sistémico, ni siquiera una coordinación normativa) anteriores existe sin embargo una migración de ideas constitucionales entre ordenamientos jurídicos separados. No se trata de lo que tradicionalmente se considera Derecho comparado porque esta migración se asienta en la convicción de que existe una «base común de comprensión o afinidad» en la situación de que se trate de modo que puedan tomarse planteamientos o soluciones ajenas como ideas con una gran autoridad persuasiva.

Ahora bien, estas situaciones de pluralismo, es obvio, responden a una geometría variable pues son muy diversas entre sí en al menos tres sentido: el competencial, el institucional y el territorial.

La entidad de las competencias atribuidas determinará lugares metaconstitucionales de intensidad variable. Cuanto más importantes y numerosas sean sus competencias, mayor necesidad práctica se tendrá de adoptar un discurso constitucional y mayor entidad tendrá la metaconstitución que lo regule. De este modo un lugar metaconstitucional que tenga atribuidas competencias de supervisión respecto a la garantía de una cuestión tan constitucional como los derechos fundamentales, desarrollará un discurso constitucional mucho más intenso que otros lugares de mero encuentro, por ejemplo, cultural.

Igualmente la estructura institucional condicionará la entidad del discurso metaconstitucional empleado. Si tal estructura se aproxima a las formas de división de poderes estatales, la necesidad del discurso será mucho mayor que si su estructura institucional responde a las tradicionales fórmulas de las organizaciones internacionales. Dicho en otros términos, si un sistema de protección de derechos (sea general o sea referido a derechos concretos) supone la creación de una instancia jurisdiccional o cuasijurisdiccional a la que puedan acceder directamente los ciudadanos, inevitablemente desarrollará un discurso constitucional mucho más intenso que una organización internacional de protección de derechos asentada sobre la igual representación de los Estados sin posibilidad de quejas individuales ante instancias independientes de resolución de reclamaciones fundadas en los derechos reconocidos en sus metaconstituciones.

Finalmente la geometría variable es clara desde una perspectiva territorial. Los niveles territoriales en los que se produce la aparición de lugares metaconstitucionales no son determinables a priori. Es evidente que determinadas organizaciones cumplen los requisitos desbrozados en el ámbito universal y en el regional. Pero pueden tener ámbitos territoriales de alcance muy variable. Algunos se situarán en una suerte de ámbito intermedio entre ambos; otros se situarán por debajo del ámbito propiamente continental (piénsese en las fórmulas regionales de integración latinoamericana); otras serán cooperaciones reforzadas en el seno de una organización internacional preexistente (piénsese en esta fórmula en la Unión Europea o en las fórmulas de opting out o en la distinta vinculación de los Estados europeos al CEDH según sean parte 
o no de los diferentes protocolos adicionales sustantivos al mismo) y, finalmente, otras pueden derivar de otros factores como puede ser la cercanía física (países fronterizos) o cultural.

De este modo podemos plantear la existencia de normas jurídicas reguladoras de lugares de ejercicio del poder que tienen dos de las tres características definidoras de la idea de constitución. Se trata de normas, en su propio ámbito, supremas y tienen por objeto una peculiar regulación de la división de poderes y un, también especial, reconocimiento de derechos a los ciudadanos. Resta por atribuir el último de los elementos. ¿Dónde está el poder constituyente? En este punto es donde más clara es la necesidad de quitarse las lentes estatales y dónde las dificultades de traducción son más nítidas.

El poder creador de estas normas constitucionales incompletas se asentaría no en un pueblo movilizado sino en la concurrencia de tres elementos definidores de una nueva forma de poder constituyente $o$, si se quiere, metaconstituyente.

En primer lugar estos centros de poder requieren algún tipo de fundamento constitucional nacional. Esto es tales centros de poder existen porque los acepta como tales expresa o tácitamente la propia constitución nacional mediante diferentes cláusulas de apertura. Lo característico es que tal fundamento es el resultado no de una concreta constitución, sino el sumatorio de todas las constituciones nacionales intervinientes en el pluralismo constitucional concreto.

En segundo lugar, el poder constituyente ya no es lo que era. No es la fuerza brutal de un pueblo movilizado (las más de las veces violentamente) que procede a destruir un orden político (USA) o el orden político y el socioeconómico (Europa). Es otro tipo de poder más suave, de naturaleza evolutiva y profundamente pragmática. Un poder constituyente que no crea la constitución sino que la permite crecer matizando o corrigiendo sus excesos. Un poder que no aparece en determinados momentos revolucionarios, sino en pequeños hitos en el camino constitucional. Un poder que no parte del acto deconstituyente (BEAUD) típico de la historia europea postrevolucionaria; sino de una continua adaptación al tiempo. En fin un poder constituyente de resabios británicos y no de naturaleza continental.

En tercer lugar, esta nueva forma de entender el poder constituyente implica una nueva forma de entender su legitimación que ya no es la democrática expresada en la movilización popular. Esta nueva forma de legitimación se asienta, por un lado, en todos los pueblos de cada uno de los Estados que en su movilización constituyente originaria introdujeron cláusulas de apertura a otros órdenes normativos y, por el otro, en el silencioso, continuo y pacífico acto de aceptación o reconocimiento de la evolución y de los contenidos constitucionales realizados en el seno de los distintos lugares metaconstitucionales. Encontramos así que el poder metaconstituyente se asienta, como probablemente no podía ser de otra forma; en legitimidad constitucional de origen, creación de un discurso constitucional propio a la manera de un poder constituyente evolutivo y reconocimiento pacífico por los Estados. 
De este modo, cuanto mayor sea la legitimidad conferida por la Constitución nacional, más intenso sea el discurso constitucional utilizado y mayor sea el grado de aceptación estatal del mismo, más importancia y entidad tendrá el lugar metaconstitucional y su norma/s fundante/s. No prescindimos de la noción de Estado ni, en concreto, de la de Constitución estatal. El nuevo orden constitucional surgido procede en gran medida, aunque nos sea la fuente única, de la constitución estatal en la que podemos encontrar su legitimidad de origen. Pero, también en ella encontraremos su legitimidad de sentido. Efectivamente, la creación o reconocimiento de metaconstituciones se produce en un necesario contexto de homogeneidad constitucional básica. Esto es no puede ocurrir que la Constitución nacional reconozca un discurso constitucional que se aparte de los elementos estructurales básicos que la inspiran. Entre la metaconstitución supraestatal y la metaconstitución estatal debe existir una continuidad de sentido sustancial. Y cuanto mayor sea el nivel de interacción y complementariedad entre ellas (lo que en otros términos significa decir cuanto mayor y más intenso sea la integración en una instancia supraestatal por la importancia cualitativa y/o cualitativa de las competencias atribuidas) mayor deberá ser la homogeneidad entre los dos lugares metaconstitucionales.

El Estado (y su Constitución) ocupa un lugar central. No un lugar exclusivo como el del Siglo XIX, pero sí un lugar central en la medida en que es cada ente estatal (a través de su Constitución) el que determina cuál es su posición en el entramado así creado. El reconocimiento de otros lugares de ejercicio del poder, el de las aspiraciones a norma suprema de sus textos fundantes y por tanto el grado de limitación de la propia constitución estatal es el resultado de la propia norma constitucional estatal y de las decisiones de los actores constitucionales principales de cada Estado.

El mejor modo para describir esta situación es, para nosotros, el de una red de constituciones interactuando permanentemente entre sí. Esta opción se nutre de construcciones extraordinariamente útiles como la de PERNICE (y F. MAYER) respecto al constitucionalismo multinivel. Si no aceptamos esta posición es por la referencia a niveles que por una parte parece (aunque sea sólo intuitivamente) reconducir a las ideas de jerarquía (superioridad del nivel superior sobre el inferior) y porque, como se ha sostenido, no resulta sencillo establecer niveles territoriales homogéneos en la integración supraestatal (ni en las transformaciones infraestatales). Nuestra posición pues se acerca más a la de los autores alemanes citados cuando utilizan el término, más próximo, de liga de constituciones o de Verfassungverbindung y que ha señalado recientemente VON BOGDANDY va tomando cuerpo cada vez más como una red en el sentido defendido en este texto.

\section{UNA RED GLOBAL DE ELEMENTOS CONSTITUCIONALES}

Una red de constituciones incompletas es el reflejo de un escenario de pluralismo constitucional (por tanto sin jerarquía entre las distintas constitu- 
ciones) en el que cada nudo representa un lugar metaconstitucional con su correspondiente metaconstitución que interaccionan entre sí en virtud del grado de reconocimiento mutuo correspondiente al nivel de homogeneidad mínima existente. Las formas de interacción serán variables según qué tipo de normas regulen las relaciones entre sí. Normas que, a su vez, pueden ser expresas o no. En la base de la red encontraremos, obviamente, la Constitución nacional en cuanto, como se ha apuntado, fuente de legitimidad y de sentido de la red. Si bien, la posición que ocupen las diferentes constituciones nacionales puede ser diferente en virtud de su grado de apertura a esa red, de modo que, incluso, pueden identificarse supuestos de autoexclusión de la red de constituciones.

La figura 1 muestra el modelo más sencillo de red. Tomando como referencia la Constitución española nos encontraríamos una red compuesta por tres nudos correspondientes a tres lugares constitucionales (España, Unión Europea y Consejo de Europa). El nudo estatal estaría conectado con el nudo UE a través del art. 93 de la Constitución que junto a las cláusulas europeas del resto de Constituciones de los Estados miembros son el origen constitucional de la propia UE, pero que han de complementarse con el proceso de constitucionalización de la Unión y con la pacífica aceptación del mismo. Dada la importancia cualitativa y cuantitativa de la integración realizada a través de la Unión Europea la homogeneidad constitucional entre ambas es muy alta y se representa gráficamente en el art. 2 del Tratado de la Unión en la redacción de Lisboa (antiguo art. 6 UE) y en los límites constitucionales implícitos a la integración (Declaración del Tribunal Constitucional español 1/2004 o más claramente el art. 23 Ley Fundamental de Bonn).

La vinculación con el otro nudo de la red (el Consejo de Europa y en particular el discurso constitucional realizado por el TEDH en la interpretación del Convenio de Roma) se produce de múltiples formas (UGARTEMENDÍA), pero de manera evidente a través del art. $96 \mathrm{CE}$ (sistema monista de recepción de tratados internacionales) y, sobre todo, mediante el uso por los actores constitucionales del art. 10.2 CE (SÁiz ARNÁIz). De nuevo la interacción entre ambos nudos es extraordinariamente intensa (aunque distinta de la producida con la UE) dada la importancia cualitativa de las competencias atribuidas que suponen la creación de un nuevo orden jurídico europeo de los derechos fundamentales. La homogeneidad constitucional básica está razonablemente asegurada al tener las mismas fuentes inspiradoras de los derechos fundamentales de la tradición constitucional europea, pero se ve reforzada por el examen previo al ingreso en el Consejo de Europa de las condiciones de respeto a los derechos fundamentales y la democracia en todo Estado aspirante.

Finalmente los dos nudos supraestatales estarán también vinculados entre sí. Lo curioso, en este caso, es que esta vinculación no implica el establecimiento de relaciones jurídicas formales. Efectivamente, pese a que así lo ordene el Tratado de Lisboa, la UE todavía no es parte del Consejo de Europa, 
ni está sometido a la jurisdicción del TEDH. Pero ello no ha impedido la generación de una línea de interacción extraordianriamente fructífera entre ambos tribunales, una relación de enriquecimiento-mutuo que ha significado la asunción de la jurisprudencia de Estrasburgo por parte de las autoridades de la Unión al mismo tiempo que el derecho de la Unión ha servido como parámetro esencial al TEDH para decidir el contenido de los derechos $\mathrm{y}$, sobre todo, para apreciar o no la existencia de un consenso europeo en las cuestiones debatidas. Las cláusulas de homogeneidad entre ambas son también claras. $\mathrm{Al}$ art. 2 TUE deberíamos unir las cláusulas interpretativas de los arts. 52.3 y 53 de la Carta de Derechos fundamentales de la UE de modo que está clara la aceptación de un común sustrato normativo en materia de derechos fundamentales. Igualmente, como aseguramiento de la homogeneidad constitucional básica podríamos entender el requisito no escrito de que el ingreso de un Estado miembro nuevo en la UE requiere del previo sello de calidad democrática y de respeto a los derechos que sólo la pertenencia al Consejo de Europa puede conferir.

FIGURA 1: CONSTITUCIÓN RED ESTADO EUROPEO

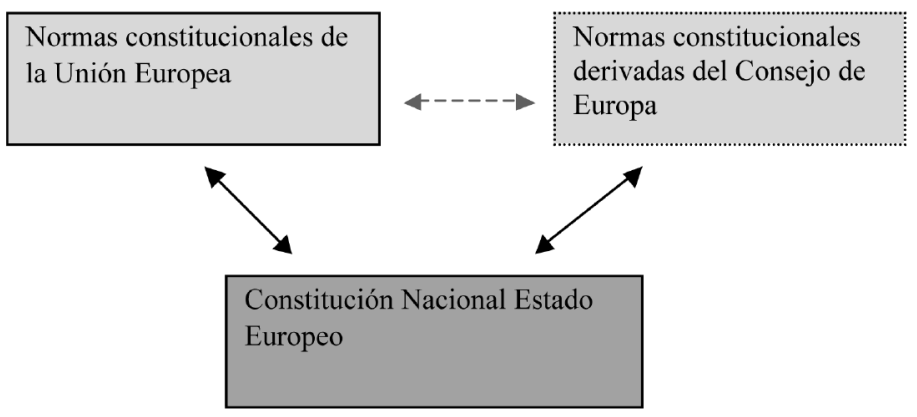

En la figura 2 la red tomará forma más clara si introducimos otros lugares como Estados miembros de la UE y del Consejo de Europa. La vinculación con ambos lugares se asentará en sus propias normas 
constitucionales. Debe notarse en este modelo la aparición de relaciones indirectas entre las Constituciones nacionales que en virtud de su doble pertenencia a ambas organizaciones sufrirán también un inevitable proceso de acercamiento entre sí.

FIGURA 2: CONSTITUCIÓN RED DE DOS ESTADOS EUROPEOS

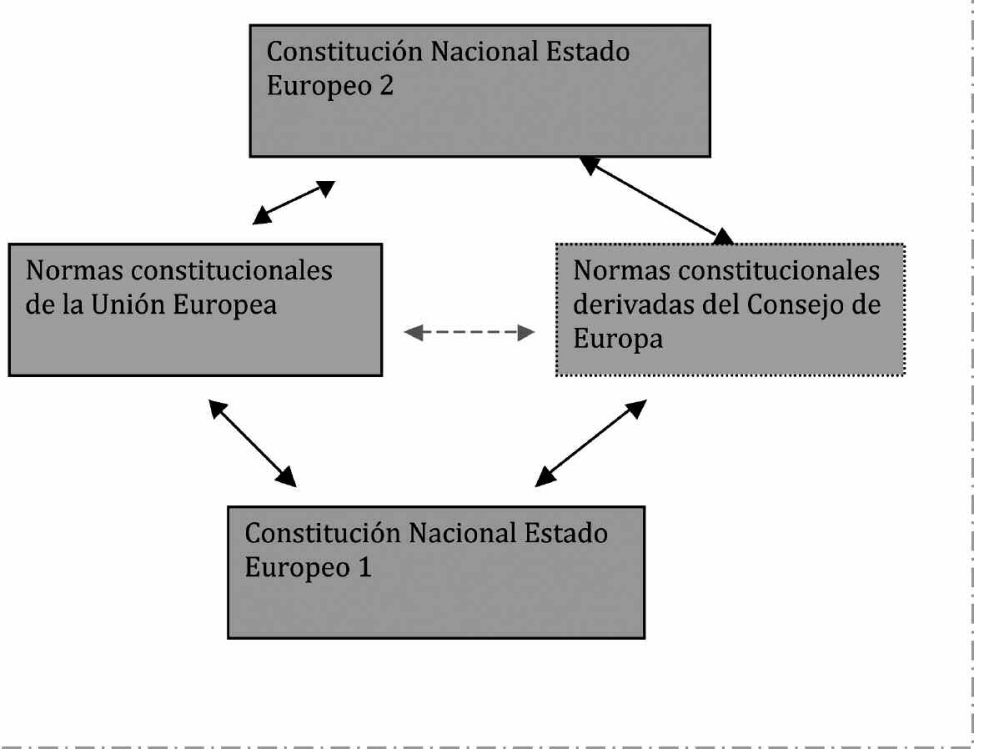

La figura 3 mostraría un supuesto de pertenencia al Consejo de Europa y no a la Unión Europea. Aquí destacaríamos la existencia de interconexiones indirectas no sólo con los Estados miembros del Consejo de Europa, sino también con la propia UE (de la que no se es parte) pero que ejerce una influencia indirecta a través del Consejo de Europa y de los Estados miembros de las dos organizaciones. 
FIGURA 3: CONSTITUCIÓN RED DE DOS ESTADOS EUROPEOS (uno de ellos que no es parte de la UE)

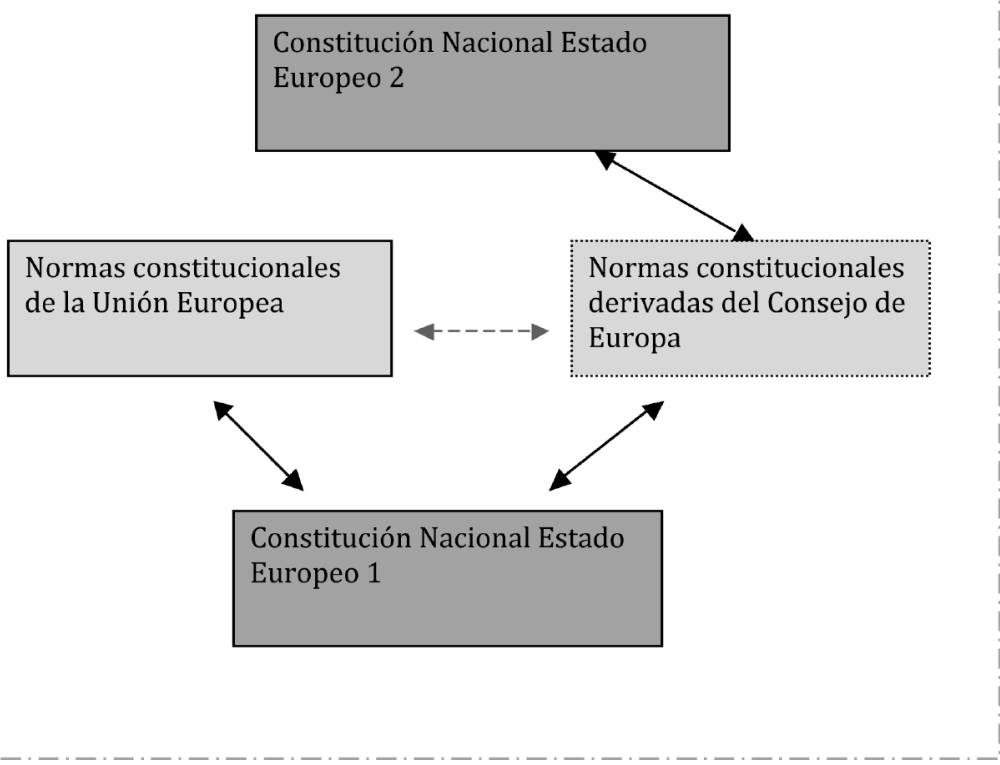

Las figuras 4 y 5 complican más el modelo pero responden a la misma lógica. En ambas asumimos la existencia de lugares metaconstitucionales de ámbito suprarregional. En la figura 4 aceptamos que buena parte de la actividad de Naciones Unidas, en especial la vinculada a la protección de derechos fundamentales, es parte de ejercicio de un discurso constitucional y que, por ello, produce normas jurídicas de naturaleza metaconstitucional que pasan a interactuar con el resto de normas metaconstitucionales a través de la red. En la figura 5 aceptamos que otros textos de ámbito internacional (vinculados o no a Naciones Unidas) pueden acabar desarrollando discursos constitucionales sustantivos (piénsese en toda la cuestión relativa a la persecución de delitos contra la humanidad). 
De nuevo tendremos relaciones de estos lugares con los Estados a través de las cláusulas de apertura al orden internacional de sus constituciones sea con carácter general o sea con carácter específico para derechos fundamentales o sea por cualquier otro instrumento (debe notarse en España, por ejemplo, que la ratificación del tratado por el que se crea la Corte Penal Internacional fue tramitada de acuerdo con el art. $93 \mathrm{CE}$ ).

La conexión en ambas figuras será, posiblemente, menos intensa que la establecida con lugares metaconstitucionales más cercanos y más importantes cualitativa y cuantitativamente. Del mismo modo, también será más débil el reconocimiento estatal de la naturaleza constitucional del discurso constitucional $^{1}$. Menor intensidad del discurso implica menor intensidad de las relaciones y menor necesidad de homogeneidad constitucional básica compartida por lo que, figura 6, podríamos incorporar al modelo a Estados no miembros de la integración europea enriqueciendo las formas de relación entre sí en todos los lugares constitucionales.

Debe notarse que las relaciones entre los diferentes lugares metaconstitcuionales tendrán diferente intensidad y naturaleza. Los distintos colores y formas de línea utilizados en las figuras no son caprichosos. Pretenden poner de manifiesto que las formas de relación son variables y denotan interacciones más o menos poderosas en función del grado de reconocimiento de las metaconstituciones entre sí. Formas de relación que a menudo no son jurídico-formales, pero no por ello dejan de ser igualmente eficaces y condicionantes del propio discurso constitucional. Así, no es extraño comprobar cómo determinados contenidos constitucionales desarrollados en un lugar metaconstitucional supranacional están condicionados por los actos de otro del que no son, formalmente, parte. Así, por ejemplo, es habitual que el TEDH se apoye en el Alto Comisionado de Naciones Unidas contra la tortura a la hora de establecer si la extradición de un extranjero detenido en alguno de los Estados miembros es o no conforme con la prohibición de tratos inhumanos o degradantes. O pueden encontrarse supuestos en los que la interpretación del Pacto Internacional de Derechos Civiles y Políticos tome como referencia la ratificación o no del Estado afectado del CEDH para poder establecer cuáles son las obligaciones realmente asumidas. $\mathrm{O}$, en fin, puede también ocurrir, que un lugar metaconstitucional asuma un nivel de protección de los derechos necesariamente superior al previsto en unos tratados de los que no necesariamente forma parte en una suerte de remisión unilateral a los mismos y a lo previsto en la Constitución de los Estados miembros o, por último, se obligue a realizar una interpretación armoniosa del contenido de los

\footnotetext{
${ }^{1}$ Muy gráficamente podemos comparar el reconocimiento, y con ello, el respeto a las decisiones del TEDH (en cuanto órgano garante del CEDH) frente al muy inferior reconocimiento y respeto dispensado por las autoridades nacionales (muy en especial las judiciales y constitucionales) al Comité de Derechos Humanos de Naciones Unidas (aunque sea un órgano garante del PIDCP para quienes hayan ratificado el protocolo facultativo anexo a éste).
} 
derechos propios con el de los derechos incluidos en las constituciones de los Estados miembros (arts. 52.2.3 y 53 CDFUE).

FIGURA 4: CONSTITUCIÓN RED DE DOS ESTADO EUROPEOS CON RECONOCIMIENTO DE UN LUGAR CONSTITUCIONAL INTERNACIONAL

\section{Constitución Nacional Estado Europeo 2}

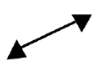

Normas constitucionales de la Unión Europea
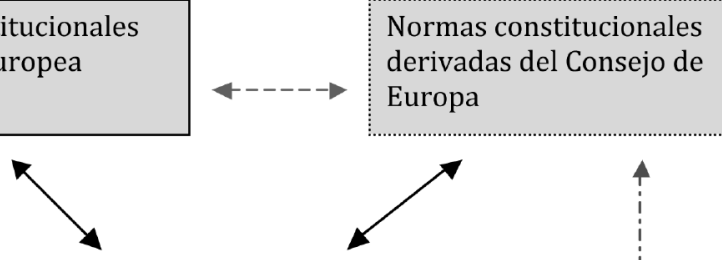

Constitución Nacional Estado Europeo 1

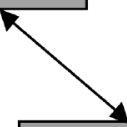

Normas constitucionales derivadas de NN UU Carta de Naciones Unidas, Declaración, Pactos de Derechos 
FIGURA 5: CONSTITUCIÓN RED DE

DOS ESTADO EUROPEOS CON

RECONOCIMIENTO DE VARIOS

LUGARES CONSTITUCIONALES

INTERNACIONALES

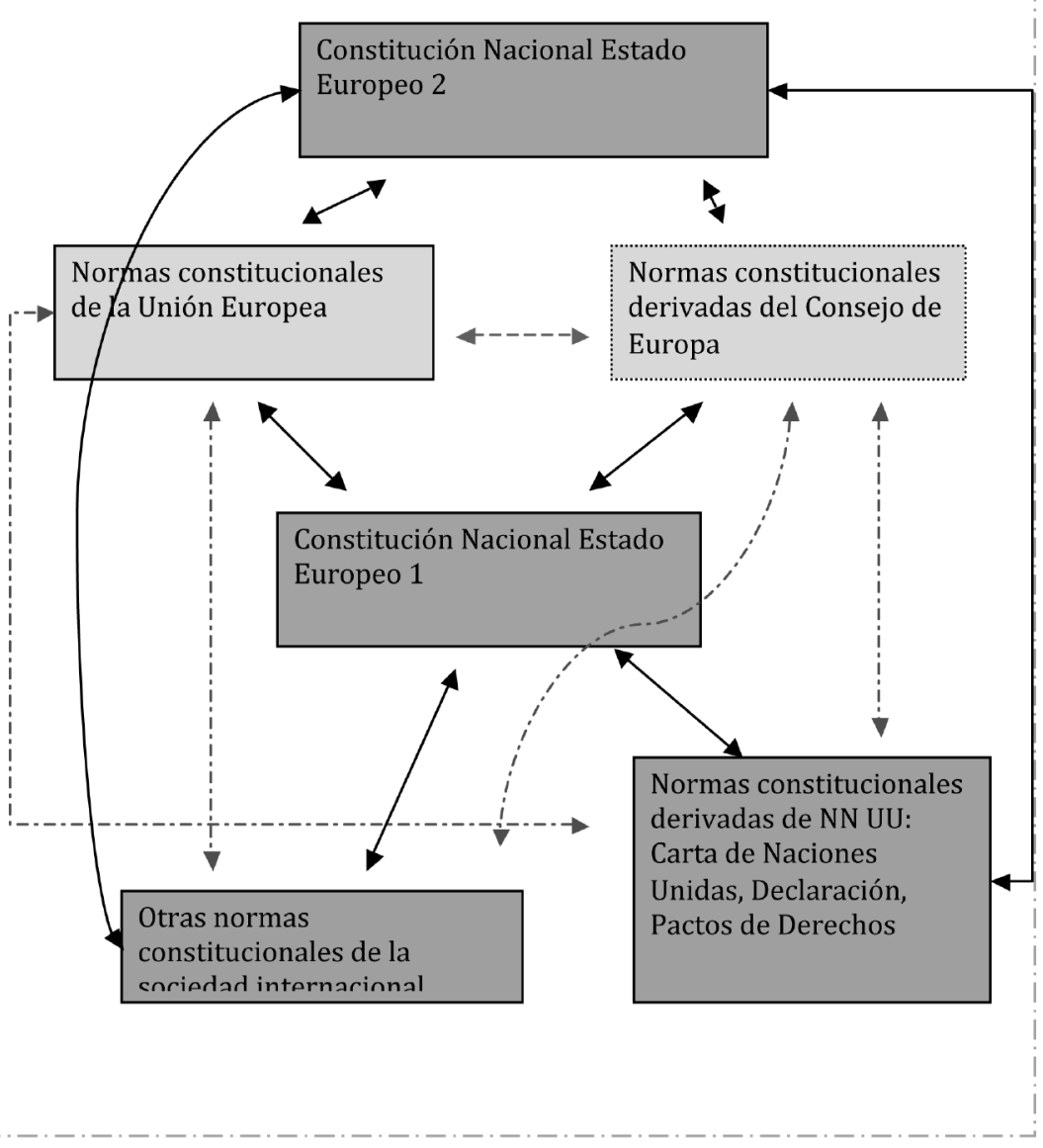




\begin{tabular}{|l|}
\hline FIGURA 6: CONSTITUCIÓN RED DE \\
DOS ESTADOS CON \\
RECONOCIMIENTO DE VARIOS \\
LUGARES CONSTITUCIONALES \\
REGIONALES E INTERNACIONALES \\
\end{tabular}

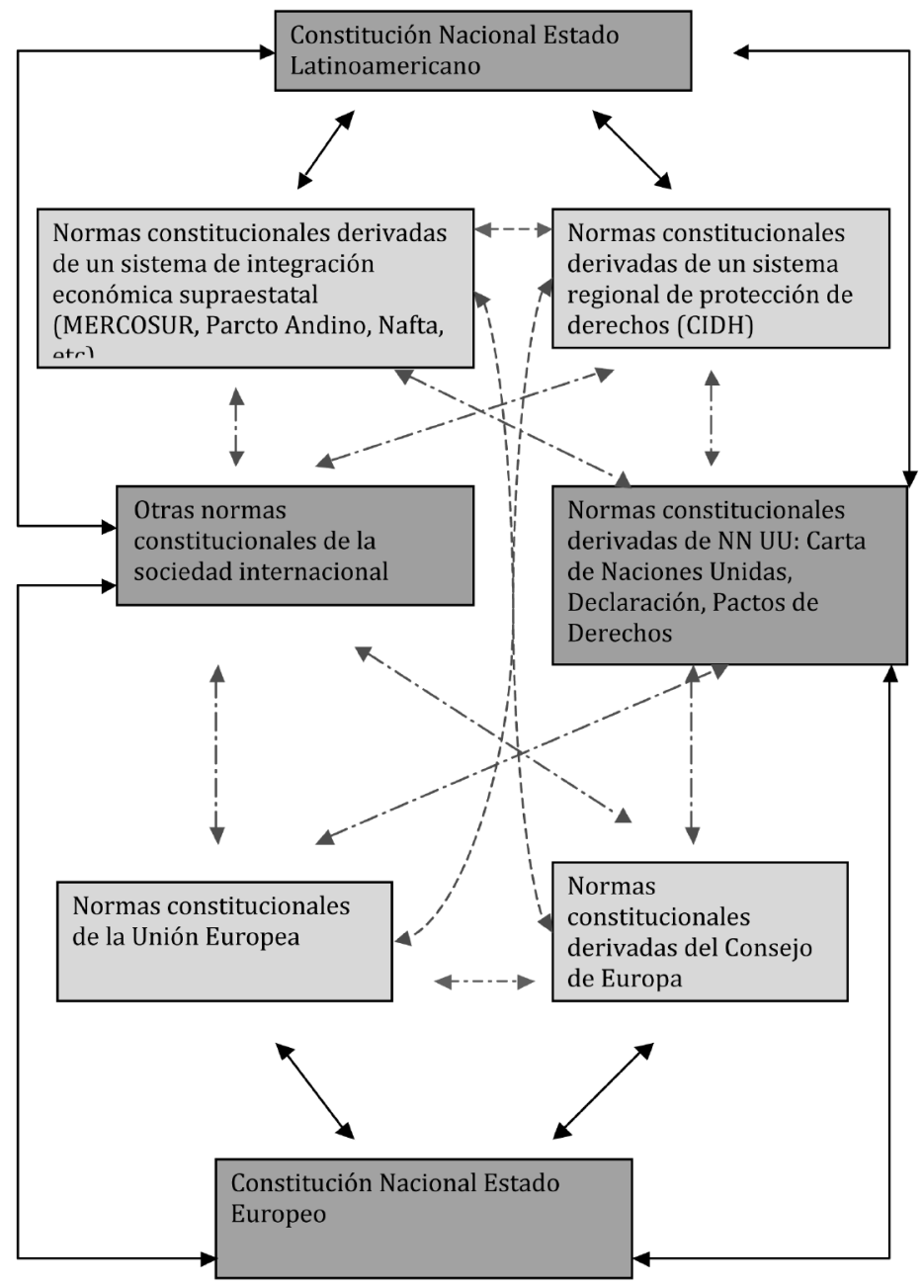


En definitiva lo que se pretende poner de manifiesto con este modelo (o cualquier otro que trate de reflejar la realidad constitucional actual) es que se ha roto la unidad documental de la Constitución. Igual que se ha roto la unidad de ejercicio del poder en manos del Estado. Si la realidad de nuestro tiempo muestra que el poder se ejerce a través de una red interconectada de centros de decisión heterogéneos, no queda más remedio que tratar de teorizar una red de constituciones que limite desde la óptica del constitucionalismo esa nueva forma de ejercicio del poder.

La ruptura de la unidad documental no significa necesariamente la ruptura de la unidad conceptual. La red de constituciones conferirá respuestas a los problemas que resolvía antes por sí sola la constitución nacional. Según el lugar de ejercicio del poder será una parte de esa red la que confiera las respuestas requeridas. Cuando ese ejercicio implique la participación de varios centros de poder, será la red de interacciones la que confiera una solución constitucional. La coherencia interna de la red vendrá asegurada por la existencia de una homogeneidad básica que habrá de ser más intensa cuanto mayores puedan ser los problemas de coherencia, esto es, cuanto mayor sea la entidad del poder (y por ello del discurso constitucional) ejercido.

Sin embargo, resta un problema por ser afrontado. Cómo resolver aquellos supuestos en los que los distintos lugares metaconstitucionales desarrollen discursos contradictorios. La asunción de un modelo de pluralismo de constituciones supone asumir que no hay una instancia superior de resolución de conflictos. Ni siquiera permite, conceptualmente, asumir una suerte de jerarquía inversa a favor de la Constitución estatal. Y no lo permite porque ello supondría volver a caer en el error de pensar que, a la postre, existe un lugar fundacional de ejercicio del poder, cosa excluida en el enfoque adoptado en estas páginas. Es este problema el que las instancias nacionales de defensa de la Constitución en Europa no parecen haber podido resolver satisfactoriamente como demuestra GONZÁLEZ PASCUAL respecto al Tribunal Constitucional federal alemán en cuanto la más significativa de ellas.

\section{LA RESOLUCIÓN DE CONFLICTOS EN LA RED CONSTITU- CIONAL}

El pluralismo constitucional no implica la creación de una situación anárquica en la aplicación del Derecho. El esquema de funcionamiento habitual en un escenario de pluralismo constitucional es la existencia de una distribución competencial clara en la que cada ordenamiento asume el ejercicio y la resolución de controversias en su propio ámbito de actuación. En este ámbito habrá siempre sólo un tipo de instancias competentes con su propio sistema jerárquico que resuelve cualquier duda de interpretación o de aplicación del derecho con independencia de que sea un sistema nacional clásico (con su pirámide judicial claramente establecida), un sistema internacional clásico (con 
sistema de paneles, de arbitraje o de tribunales permanentes) o sistemas nuevos (la peculiar estructura judicial de la UE con sus órganos centralizados y el poder judicial de los Estados miembros).

Por tanto, no hay conflicto permanente entre ordenamientos. Cada uno tiene su propio ámbito competencial con los órganos encargados de resolver en última instancia.

Esta distribución a partir del principio de competencia presenta inevitablemente dos aspectos difícilmente resoluble referidos a los márgenes de actuación de cada ente: aquellos puntos en los que se producen intersecciones entre ordenamientos.

El primero, detectado con toda claridad a partir de la decisión Maastricht (MACCORMICK -1995-; WeILER/HALTERN -1996-), tiene que ver con la determinación en última instancia de la amplitud de las respectivas competencias en las que se mueve cada ordenamiento. $\mathrm{O}$ dicho en otros términos la cuestión radica en determinar a quién corresponde la titularidad de las competencias cuando éstas sean el propio objeto de controversia. Los sistemas federales sitúan esta Judicial Kompetenz-Kompetenz en el órgano jurisdiccional supremo del Estado federal o en un Tribunal Constitucional que, por definición, es un órgano federal. Esto es, asignan la última palabra a un órgano de la entidad territorial que engloba a todas aquellas que se integran en su seno (lo que puede incluir, como es bien sabido en España, no sólo a las entidades regionales, sino también a las locales). Esta solución no es posible en un escenario de pluralismo constitucional.

En efecto, en este tipo de escenarios el alcance de la propia competencia la determina el órgano encargado de resolver en última instancia todos los conflictos planteados en el seno del propio ordenamiento. De este modo es perfectamente lógica la aparición de dos narrativas (MADURO, 2003) contradictorias. Se puede, así, producir que, por ejemplo, el TJUE considere que la determinación de las competencias asumidas por la Unión sea objeto de su competencia última en cuanto guardián último de los tratados fundacionales. Pero también es igualmente lógico que, por ejemplo, el TCF considere, como viene haciendo desde su famosa decisión sobre el Tratado de Maastricht hasta la más reciente sobre el Tratado de Lisboa, que la determinación de las competencias transferidas a la Unión le puede corresponder a él en cuanto guardián último del ordenamiento jurídico alemán lo que, lógicamente, incluye la delimitación última de las sucesivas leyes de ratificación de los tratados fundacionales de la Unión Europea (argumento que mutatis mutandis podría extenderse probablemente a los restantes Estados miembros de la Unión). La solución a una eventual contradicción entre ambas jurisdicciones (la europea y la nacional) no puede resolverse con el predominio de ninguna de las dos so pena de concluir que realmente no estamos ante una situación de pluralismo constitucional entre ordenamientos a un mismo nivel sino en una situación clásica de monismo (sea de predominio del orden supraestatal, sea de predominio del orden estatal). Estamos, pues, en presencia de un primer campo de 
conflicto $^{2}$. El, en la afortunada expresión de WALKER (2003), territorio disputado entre dos ordenamientos autónomos que recaban para sí el ejercicio de la competencia controvertida y, con ello, del poder de decidir en última instancia a quién corresponde su ejercicio. Por tanto, es en el enjuiciamiento de la eventual actuación ultra vires de los diferentes poderes generadores de los distintos ordenamientos donde encontramos un primer problema no resoluble desde la aplicación del principio de competencia. Y con ello es en este campo en el que nos podemos encontrar soluciones contradictorias y no compatibles entre las diferentes máximas instancias de defensa del propio ordenamiento. Este es, pues, el primer ámbito de actuación del diálogo judicial en el sentido utilizado en estas páginas. Un ámbito donde desarrolla su plena potencialidad y en el que asume un papel distinto a nada conocido anteriormente y que lo diferencia de otras formas de comunicación judicial.

El segundo ámbito en el que el principio de competencia presenta especiales dificultades para delimitar la propia esfera de actuación es también conocido en los sistemas descentralizados en general y en España en particular. En efecto, esa rara avis en el panorama comparado constituida por la competencia contenida en el art. 149.1.1 ${ }^{\mathrm{a}}$ ha generado una más que abundante jurisprudencia y literatura especializada (véase con carácter general GONZÁLEZ PASCUAL, 2007). El problema que aquí se aborda es la existencia de competencias que por no ser reconducibles a los conceptos propios de la distribución material de poderes (sea interna, sea respecto a instancias supraestatales) son conocidas como competencias horizontales o transversales y que en el campo de la integración de ordenamientos internacionales se centran en la protección y garantía de los derechos fundamentales. Efectivamente, en esta materia todos los ordenamientos pueden incidir. Sea el ordenamiento nacional, sea el ordenamiento de la Unión Europea, sea el Derecho Regional de los derechos humanos, sea el Derecho internacional-universal de los derechos humanos, sea el Derecho internacional no específicamente dirigido a los derechos fundamentales. Se trata de situaciones en las que lo producido son superposiciones (overlappings) entre las regulaciones de distintos ordenamientos sobre un mismo objeto y no auténticos conflictos.

De nuevo lo característico frente a las fórmulas tradicionales de descentralización con reconocimiento de diversidad de declaraciones de derechos en el ámbito federal y en el estatal es la ausencia de una entidad jurisdiccional suprema de decisión (la del ámbito de decisión territorial más amplio) capaz de resolver la cuestión, parafraseando al CJ JACKSON, no por ser infalible sino simplemente por ser la última.

${ }^{2}$ Nótese que parecida situación se produce si lo que se discute es la intensidad de la supervisión de un tribunal internacional de derechos. Tanto en los conflictos entre el TEDH y los tribunales constitcuionales como entre éstos y por ejemplo el Comité de Derechos Humanos de NN UU, el primer punto de la discusión es una cuestión competencial relativa a la extensión de los poderes de supervisión de los órganos supraestatales. 
Estamos, pues, ante un segundo territorio disputado: el de la protección y garantía de los derechos fundamentales. Un campo en que diversos entes, con distintos textos de referencia y distintas formas de relacionarse entre sí, carecen, por definición, de una autoridad última de determinación del contenido de los mismos. La posibilidad de que lleguen a definiciones no coincidentes generadoras de obligaciones incompatibles entre sí es bastante más que probable si tales instancias pretenden asumir tal competencia interpretativa. Es, por tanto, este segundo campo donde también la idea de diálogo judicial como nuevo concepto cobra su pleno sentido.

En tales situaciones, como hemos tratado de demostrar en otro lugar, la salida habitual es el establecimiento de un diálogo entre las instancias judiciales llamadas a aplicar su propia metaconstitución teniendo en cuenta el resto de metaconstituciones presentes en la red. Esta obligación de tener en cuenta (to take into account) las resoluciones judiciales tendrá tanta intensidad como tenga la imbricación entre ordenamientos diseñada en las constituciones en conflicto. Pero acabará o proponiendo una situación mutuamente aceptable para los dos entes (una interpretación mutuamente conforme, una interpretación compatible, una prioridad en la decisión, una aceptación de la decisión ajena, etc) o constatando un conflicto irresoluble. En el primer caso estaremos ante un diálogo judicial con éxito que tras un cierto tiempo resuelve el conflicto; en el segundo caso daremos origen a la negociación política entre los lugares metaconstitucionales en conflicto para modificar sus propias metaconstituciones y resolver el enfrentamiento.

Esta apelación a la negociación política se inspira no sólo en la práctica de la integración regional o internacional sino en la solución conferida en algunos Estados al conflicto entre ley y derechos fundamentales. En concreto nos referimos a la solución conferida a tal conflicto en la Human Rights Act británica. La singularidad del sistema británico en este punto se encuentra en que un eventual conflicto entre una norma soberana (en el sentido británico del término, esto es aprobada por el King in Parliament) y uno de los derechos contenidos en la HRA (que reproducen el CEDH) no se resuelve automáticamente con la prevalencia de una u otra. El conflicto, una vez detectado judicialmente, origina una proceso cooperativo entre ejecutivo y legislativo tendente a la modificación del ordenamiento para eliminar la incompatibilidad detectada.

Algo similar ocurre, a nuestro juicio, en la práctica de la integración regional. En ocasiones el diálogo jurisdiccional no puede resolver los conflictos porque carece de los poderes suficientes para hacerlo y sólo puede constatar la existencia de una incompatibilidad. En estos casos se utiliza la negociación. Entendida como la conversación entre los actores jurídico-constitucionales de los diferentes lugares en conflicto tendentes a resolverlo mediante la modificación de las normas generadoras del mismo. La historia de la integración europea muestra estas negociaciones de manera evidente; en ocasiones previniendo conflictos y en otras respondiendo a los ya declarados. La infinidad 
de protocolos adicionales aprobados para respetar identidades constitucionales específicas; la presencia del respeto a la singularidad consittucional de cada Estado miembro en el art. 2 de actual tratado, las frecuentes reformas constitucionales de los Estados con motivo de la ratificación de tratados europeos, no son más que formas cooperativas de prevención y resolución de conflictos.

Sea como fuere tanto en un diálogo judicial exitoso como en una negociación política un principio básico del Estado de Derecho sufre gravemente. El principio de seguridad jurídica y en particular el de previsibilidad del Derecho que nutre a aquél. Efectivamente en los tipos de situaciones descritas no habrá ninguna solución previsible, es más según acudamos a unas u otras instancias recibiremos, por definición, soluciones contradictorias sin ninguna posibilidad de resolver el asunto en una instancia superior común. ¿puede tal situación tolerarse desde la seguridad jurídica? Una respuesta clásica dirá que en absoluto. Una respuesta adaptada a los tiempos sostendrá que mientras esa situación no se eternice en el tiempo y acabe encontrando una salida razonablemente previsible será tolerable desde el punto de vista de la seguridad jurídica.

En este sentido, y con ello queremos concluir, es particularmente interesante la sentencia del TEDH Nejdet Sahin \& Perihan Sahim contra Turquía, de 20 de octubre de 2011. El asunto se centra en la resolución de una reclamación contra las autoridades es la existencia de dos jurisprudencias contradictorias en lo que podríamos denominar dos salas del mismo tribunal supremo (realmente se trata de dos Tribunales Supremos pues en Turquía hay cuatro tribunales supremos, uno por jurisdicción). El problema planteado es que un mismo supuesto de hecho (la muerte en accidente de avión de diversas personas) es resuelto de manera contradictoria a los efectos del reconocimiento de pensiones especiales a los familiares. Así en un caso, el de los recurrentes, se niega tal reconocimiento por el Tribunal Supremo Militar, mientras que en los restantes se otorga tal reconocimiento por el Tribunal Supremo Administrativo. En ausencia de una instancia de unificación de las decisiones, el TEDH se encuentra ante un supuesto en el que dos tribunales supremos son igualmente competentes y adoptan decisiones contradictorias entre sí. Los reclamantes asientan sus alegaciones en el art. 6, pero realmente el problema es si la presencia de dos jurisprudencias contradictorias para el mismo caso es lesivo del principio de seguridad jurídica. Como puede observarse el caso reproduce con una peculiaridad legislativa interna la situación de concurrencia perfecta entre instancias jurisdiccionales de protección de derechos todas ellas competentes y últimas que llegan a conclusiones distintas. Por ello no es de extrañar que más allá de la importancia del caso, la Gran Sala se dividiera ( 10 a 7 ) en dos grupos con importantes magistrados en cada bando.

La mayoría entendió que una situación como ésta es tolerable siempre y cuando no sea permanente en el tiempo y no afecte a cuestiones particular- 
mente importantes, por un lado, y que existan mecanismos para resolver el problema en el futuro, por el otro.

Para la minoría la situación es inaceptable desde el punto de vista de la seguridad jurídica y denota un defectuoso funcionamiento estructural de los sistemas turcos de resolución de conflictos jurisdiccionales. La lesión al art. 6 es, para la minoría, obvia pues lo contrario convertiría a la Justicia en una lotería (sic) en la que la resolución en un sentido u otro del caso depende solo de qué juez sea el encargado de resolverlo.

La fuerte división, la importancia de los firmantes e incluso el tono agresivo en que están redactados algunos párrafos denota la importancia del debate en un asunto que, sin duda, tampoco era una cuestión esencial para la vigencia de los derechos fundamentales. Detrás de este duro y apasionante debate hay otro, más profundo que tiene que ver con la necesidad o no de adaptar a las nuevas realidades los viejos conceptos. La existencia de elementos constitucionales en una red global que lleva aparejada la concurrencia de tribunales en la protección de derechos pudiendo originar jurisprudencias contradictorias puede ser entendido como una solución aceptable en determinadas condiciones (en particular respecto a la entidad de las contradicciones y al tiempo de inseguridad) por unos, o la conversión de la justicia en una lotería, por otros.

El debate sigue abierto...

\section{BIBLIOGRAFÍA UTILIZADA}

Hemos respetado la estructura y la lógica de la exposición oral de la que procede el texto reproducido por lo que recogemos en este apartado las referencias expresamente realizadas en el mismo. Con carácter general la ponencia recoge sustancialmente, actualizando y cambiando algunos aspectos, las tesis sostenidas en nuestro trabajo La Constitución Red: un estudio sobre supraestatalidad y Constitución, IVAP, Vitoria, 2005. Igualmente se recogen algunas reflexiones suscitadas al hilo de la publicación de Pluralismo constitucional y diálogos, México, Purrúa (2012) y del artículo «XV proposiciones para una teoría general del diálogo judicial»; Revista Española de Derecho Constitucional, 95, 2012. Muchas de las cuestiones sólo apuntadas en la ponencia se desarrollan en esos tres textos así como buena parte de la bibliografía utilizada para la construcción de las tesis defendidas. Nos limitamos, por tanto, a reproducir sólo las referencias de los autores recogidas en el texto.

CRuz Villallón, P., La Constitución inédita. Estudios ante la constitucionalización de Europa. Madrid, 2004.

GARcía RocA, J., La doctrina del margen de apreciación nacional en la jurisprudencia del TEDH, Civitas, Madrid, 2009.

GonzÁlez Pascual, M. I., El Tribunal Constitucional alemán en la construcción del espacio europeo de los derechos, Civitas-Thomson, Madrid, 2010. 
Gordillo Pérez, L. I., Constitución y ordenamientos supranacionales, CEPC, Madrid, 2012.

GuTIÉRREZ GutiéRreZ, I., «Traducir derechos: la dignidad humana en el derecho constitucional de la comunidad internacional», AFDUAM, num 16, 2012.

GutiÉrRez GutiérRez, I., «Alcance de los derechos fundamentales en el nuevo constitucionalismo supranacional», en M. A. PRESNO \& I. GutiÉRREZ, La inclusión de los otros: símbolos y espacios de la multiculturalidad, Granada, 2012.

Grimm, D., «Does Europe Need a Constitution», en P. GOWAN, P. ANDERSON (ed), The Question of Europe, Londres-Nueva York, 1997.

Habermas, J., «Reply to Grimm», en P. Gowan, P. ANDERson (ed), The Question of Europe, Londres-Nueva York, 1997.

MAYER, F. C., «The European Constitution and the Courts. Adjudicating European Constitutional Law in a Multilevel System», en A. VoN BogDANDY (ed), European Integration: the New German Scholarship, Jean Monnet Working Paper, 9, 2003 (www.jeanmonnetprogram.org/papers/03).

MacCormick, N., «Beyond the Sovereign State», en The Modern Law Review, 56, 1993.

MacCormick, N., Questioning Sovereignty. Law, State and Practical Reason, Oxford, 1999.

Pernice, I., «Multilevel Constitutionalism and the Treaty of Amsterdam: European Constitution Making revisited? », en Common Market Law Review, 36, 1999.

PERnICE, I., «Multilevel constitutionalism in the European Union», en European Law Revies, vol 27, 5, 2002.

PERNICE, I., «The treaty of Lisbon: multilevel constitutionalism in action», en Columbia Journal of European Law, 15, 2009.

Pernice, I., Mayer, F. C., «De la constitution composée de l'Europe», en Revue Trimestrielle de Droit Européen, 36, 4, 2000.

Queralt JimÉNEZ, A., El Tribunal de Estrasburgo una jurisdicción internacional para la protección de los derechos fundamentales. Valencia, 2003.

QUERALT JiMÉNEZ, A., La interpretación del los derechos: del tribunal de Estrasburgo la Tribunal Constitucional, CEPC, Madrid, 2008.

RöBEn, V., Constitutionalism of Inverse Hierarchy: the Case of the European Union, Jena Monnet Working Paper, 8/03, Nueva York, 2003.

SÁIZ ARNÁIZ, A., La apertura constitucional al Derecho Internacional y Europeo de los derechos humanos. El art. 10.2 de la Constitución Española, Madrid, 1999.

Torres Pérez, A., Conflicts of rights in the European Union. A Theory of Supranational Adjudication. Oxford, Oxford University Press, 2009.

UGARTEMENDÍA ECEIZABARRENA, J. I., «Cauces de interacción e incorporación de los derechos fundamentales de la Unión Europea», en A. SÁIZ ARNÁIZ 
(dir) \& M. Zelaia Garagarza (coord.), Integración Europea y Poder Judicial, IVAP, Vitoria, 2006.

Walker, N. (ed), Sovereignty in Transition, Oxford/Pórtland, 2003.

Walker, N., The Idea of Constitutional Pluralism, EUI Working Paper, 2002/1, Florencia, 2002 (www.iue.it).

WALKER, N., «Beyond boudary disputes and basic grids. Mapping the global disorder of normative orders», en International Journal of Constitutional Law, vol 6, num 3-4, 2008.

Von Bogdandy, A., «Doctrine of Principles», en A. Von Bogdandy, A.European Integration: the New German Scholarship, Jean Monnet Working Paper, 9, 2003 (www.jeanmonnetprogram.org/papers/03).

Von Bogdandy, A., «Los principios fundamentales de la Unión Europea. Aspectos teóricos y doctrinales», en Revista General de Derecho Europeo, 22, 2010.

Weiler, J. H. H., «Does Europe Need a Constitution?. Reflections on Demos, Telos and Ethos in the German Maastricht Decision», en P. Gowan, P. ANDERSON (ed), The Question of Europe, Londres-Nueva York, 1997. 